\title{
INFORMATION SYSTEMS
}

\section{ИНФОРМАЦИОННЫЕ СИСТЕМЫ}

\section{УДК 355.01:004.056}

\author{
А.А. Перцев, А.Н. Подобрий, Ю.А. Радионова
}

\section{ПЛАНИРОВАНИЕ РАСХОДА МАТЕРИАЛОВ ДЛЯ ОБЕСПЕЧЕНИЯ ПРОИЗВОДСТВА МАШИНОСТРОИТЕЛЬНОГО ПРЕДПРИЯТИЯ}

Перцев Андрей Алексеевич, кандидат технических наук, окончил механико-математический факультет Ульяновского государственного университета. Начальник отдела ФНПЦ АО «НПО «Марс». Имеет статьи в области внедрения автоматизированной системы управления предприятием. [e-mail:mars@mv.ru].

Подобрий Александр Николаевич, кандидат технических наук, окончил механико-математический факультет УлГУ. Заместитель начальника отдела ФНПЦ АО «НПО "Марс». Имеет статьи в области внедрения автоматизированной системы управления предприятием. [e-mail: mars@mv.ru].

Радионова Юлия Александровна, кандидат технических наук, окончила механико-математический факультет УлГУ. Ведущий инженер-программист ФНПЦ АО «НПО «Марс». Имеет публикации в сфере автоматизированных систем документооборота, интеллектуальной организации хранилищ технической документации, статистической оценки поставщиков. Сфера научных интересов: электронный документооборот, архивохранилища, статистический анализ данных, системы поддержки принятия решений. [e-mail: julia-owl@mail.ru].

\section{Аннотация}

В статье представлен подход к планированию расхода материалов для обеспечения производства проектной организации, выпускающей машиностроительное оборудование, на основании статистики расхода материалов за предыдущие периоды и очередности изготовления продукции. Рассматриваются варианты использования информационных технологий планирования производственных запасов.

Предложена модель обеспечения материалами производства на основе анализа временных рядов (ВР). Описана схема исследования и структура базы данных (БД) для расчетов по разработанной модели. Детализированы этапы алгоритма анализа данных, моделирования ВР и сравнения полученных значений с тестовыми данными. Приводятся экспериментальный расчет проверки корректности построенной модели и сравнительные графики значений ВР для образцов основного и вспомогательного материалов. Программная реализация расчетов представлена в виде дополнительного модуля для интерактивного веб-ресурса и использует T-SQL для реализации.

Ключевые слова: формирование плана производства, временные ряды, критический путь, сетевой график, материал, ресурсы, проектное производство, машиностроение, мощность производства, статистика выполнения операций, посменно-суточное планирование.

doi: $10.35752 / 1991-2927-2021-1-63-34-45$ 


\section{MATERIAL SCHEDULING TO PROVIDE MANUFACTURING IN MACHINE INDUSTRY}

Andrei Alekseevich Pertsev, Candidate of Sciences in Engineering; graduated from the Faculty of Mechanics and Mathematics of Ulyanovsk State University; Head of an department at FRPC JSC 'RPA 'Mars'; an author of articles in the field of the automated enterprise management system implementation.e-mail: mars@mv.ru.

Aleksandr Nikolaevich Podobrii, Candidate of Sciences in Engineering; graduated from the Faculty of Mechanics and Mathematics of Ulyanovsk State University; Deputy Chief of a department at FRPC JSC 'RPA 'Mars'; an author of articles in the field of the automated enterprise management system implementation.e-mail: mars@mv.ru. Iuliia Aleksandrovna Radionova, Candidate of Sciences in Engineering; graduated from the Faculty of Mechanics and Mathematics of Ulyanovsk State University; Lead Programming Engineer at FRPC JSC 'RPA 'Mars'; an author of articles in the field of automated workflow systems, intelligent technical documentation storage bases and systems for statistical analysis of supplier appraisal; research interests are in the field of electronic document management, archival depositories, statistical data analysis, decision support systems.e-mail: julia-owl@mail.ru.

\section{Abstract}

The article describes an approach to the material scheduling to provide manufacturing by machinery designers. The approach is based on the statistics of material consumption over the last periods and on manufacturing sequence. The article considers information technology alternatives of production reserve planning.

The authors propose the model of material support based on a time series analysis. They describe a study scheme and a database structure for calculating by the model developed. The algorithm steps of data analysis, modeling of time series and resulted VS test values comparison are described in details. The article defines an experimental calculation to test the model validity and diagrams to compare the time series of the main and auxiliary material sample pieces. The programmed calculations are given as an additional module for interactive web resource and are implemented through T-SQL.

Keywords: manufacturing plan formation, time series, critical path, scheduling network, material, resources, project manufacturing, mechanical engineering, capacity, statistics of operations fulfilled, shiftwork scheduling.

\section{ВведенИЕ}

Основной задачей планирования подготовки производства являются определение всех видов работ, их объёмов, сроков выполнения, исполнителей, взаимоувязка по срокам выполнения всех этапов подготовки производства, определение трудовых, материальных и финансовых затрат на подготовку.

Целью планирования является максимальное сокращение сроков подготовки и освоения производства новых изделий при наименьших затратах трудовых, материальных и финансовых ресурсов. Данный вид планирования называется оперативно-производственным планированием и должен выполнять следующие функции [1]:

- объёмные расчёты по предприятию и производственным подразделениям;

- расчёты календарно-плановых нормативов организации производственного процесса;

- разработка календарных графиков запуска-выпуска изделий;

- формирование оперативных производственных заданий подразделениям предприятия;

- оперативное регулирование, учёт и контроль выполнения плановых заданий.

Оперативно-производственное планирование можно разделить на три этапа.

1. Планирование на заводском уровне заключается в распределении выпуска продукции по календарным срокам (месяцам планируемого года), а при позаказной системе - по номерам неделей. На этом этапе достигается такое распределение продукции, при котором могут быть получены, с одной стороны, наилучшие финансовые результаты, с другой - равномерная продолжительность циклов сборки. Для достижения этих целей используются: номенклатура изделий, их цена и рентабельность, трудоёмкость и станкоёмкость. Полученный таким образом объёмный план производства служит основой для разработки новых планов. На основе календарно-плановых нормативов (партий запуска, циклов и опережений) составляются производственные планы каждому цеху. В планах указываются не только модели и количество изделий, но и номера изделий, планируемых к выпуску. На основе разработанного объёмного плана производства определяются необходимые ресурсы для его выполнения (потребности в материалах, комплектующих, трудовых ресурсах и денежных средствах).

2. Планирование на межцеховом уровне заключается в том, чтобы объёмные цеховые планы превратить в подетальные. На основе календарно-плановых нормативов, спецификаций и расцеховочных ведомостей изделия расписываются по деталям. Составляется подетальный план запуска-выпуска деталей с указанием их номеров, количества в партиях, сроков их запуска и номеров выпускаемых изделий, которые ими будут укомплектованы.

3. Внутрицеховое планирование. На этом этапе подетальный план запуска-выпуска превращается в 
плановые задания на смену на каждое рабочее место (сменные задания). Этот этап условно называют ещё и оперативным планированием, поскольку именно на этом этапе вскрываются и оперативно устраняются все недостатки предыдущего планирования. Работу по планированию выполняют цеховые специалисты. Они составляют ежедневно на следующую смену для каждого рабочего сменные задания на основе следующих сведений: о наличии заготовок на складе, плана запуска-выпуска деталей, маршрутных карт и норм времени, партий запуска. При этом проверяется готовность производства к выполнению задания: наличие заготовок, состояние оборудования, наличие документации, оснастки, инструмента. При необходимости принимаются меры. Если устранить недостатки за период подготовки не удаётся, к производству планируются другие детали. После выполнения рабочим сменного задания детали или изделия предъявляются контролёру. В сменном задании делается отметка о количестве фактически изготовленных изделий или деталей.

Основной задачей при нормировании производственных запасов является определение минимального размера запаса, при котором обеспечивается непрерывность производственного процесса. Производственные запасы предприятия делятся на текущие (оборотные), подготовительные и страховые (резервные). Структура производственных запасов показана на рисунке 1.

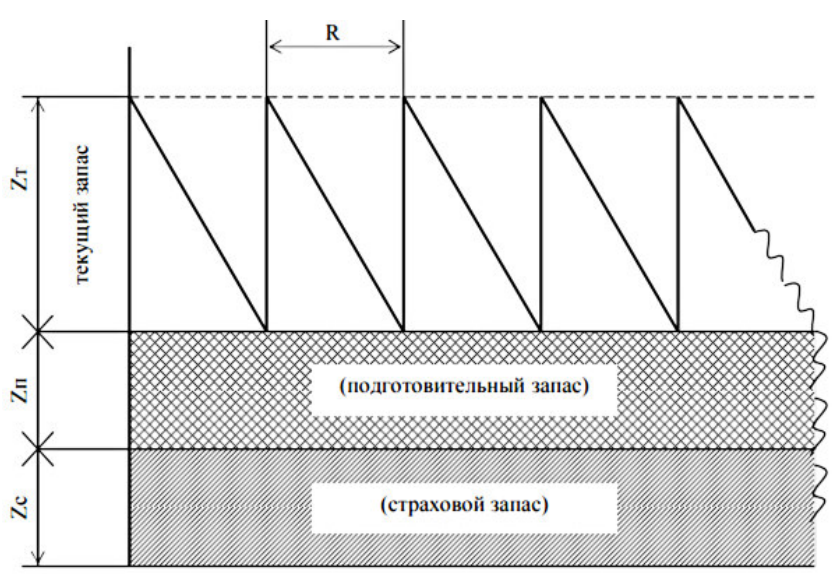

Рис. 1. Структура производственного запаса

Под текущим или оборотным запасом понимается часть общего запаса, которая систематически расходуется в производстве в период между смежными поставками материальных ресурсов (ритм поставки, $R$ ).

Максимальная величина текущего запаса равна объёму партии поставки и определяется по формуле:

$$
Z_{T, M A X}=P * R,
$$

где $P$ - среднесуточная потребность (расход) в материальном ресурсе,

$R$ - ритм поставки.
Подготовительный запас создается с целью компенсации времени, необходимого на разгрузку материалов, их приемку, размещение на складе, подготовку к выдаче в производство. Размер подготовительного запаса определяется временем подготовки:

$$
Z_{n}=P \cdot t_{n},
$$

где $t_{n}$ - время подготовки материалов.

Под страховым запасом понимается часть запаса, необходимая для обеспечения производства в случае исчерпания текущего запаса и задержки поставки очередной партии материалов. Величина страхового запаса определяется по формуле:

$$
Z_{s t r}=P \cdot \Delta R,
$$

где $\Delta R$ - среднестатистическая величина задержки поставки очередной партии материала,

$P$ - размер партии поставляемого материала.

Максимальный производственный запас определяется суммой максимального текущего, подготовительного и страхового запасов и используется для расчета площади складских помещений.

Зачастую для решения задачи оперативно-производственного планирования используют два варианта информационных технологий планирования [2, 3]:

1) внедрение MRP II (Material Requirement Planning - Планирование материальных ресурсов) и CRM (Customer Relationship Management - Управление взаимоотношениями с клиентами) совместно;

2) внедрение ERP (Enterprise Resource Planning System - Система планирования ресурсов предприятия).

MRP II [4] - это метод планирования всех ресурсов производственной компании, оперативное планирование в единицах продукции, финансовое планирование в денежных единицах с элементами моделирования. Данная программа касается ряда функций, каждая из которых связана с остальными: бизнес-планирование, планирование продаж и операций, объемно-календарное планирование, планирование потребности в материалах, планирование потребности в производственных мощностях, а также систем поддержки производственных мощностей и материальных ресурсов.

CRM [5] - это методология управления ресурсами предприятия, ориентированная на продажи и взаимоотношения с клиентами. В более общем смысле управление отдельными функциями службы продаж (sales forces) и технологии автоматизации этих функций.

Отдельное использование MRP II или CRM систем может лишь компьютеризировать и частично автоматизировать соответствующие функции на предприятии [1]. Поэтому в первом варианте предлагается их совместное использование.

Второй вариант модернизации предлагает использование более сложной концепции ERP, в основе которой лежит принцип создания общего хранилища данных. Данная система позволяет проводить производственное планирование, моделировать поток заказов и оценивать возможность их реализации в службах 
и подразделениях предприятий, управления корпоративными ресурсами.

К свойствам MRP II добавилось управление финансовыми ресурсами, маркетинг. ERP-концепция - это первая направленная на управление бизнесом, а не только производством, как MRP, концепция бизнес-планирования. Важным отличием от методологии MRP II является возможность “динамического анализа» и "динамического изменения плана» по всей цепочке планирования.

\section{1 ВидЫ РЕСуРСОВ}

Ресурсное обеспечение, составляющее важное условие осуществления любого вида хозяйственной деятельности, приобретает новое содержание в процессе управления проектами. Проектная деятельность, по сравнению с другими видами бизнеса, требует сложного набора ресурсов, инвестируемых при повышенном риске и на более длительный срок. Это предъявляет особые требования к организации ресурсного обеспечения.

Можно выделить четыре основных вида ресурсов, используемых в процессе управления проектами: кадровые ресурсы, материально-технические, оборудование и финансовые.

Люди являются наиболее очевидным ресурсом проекта. Людские ресурсы обычно классифицируются по их рабочему профилю - например, программист, инженер-механик, сварщик, контролер, заведующий отделом сбыта, инспектор. В редких случаях некоторые умения взаимозаменяемы, но при этом, как правило, теряется производительность. Наличие многих квалификаций усложняет составление календарного плана проекта.

Задержка в выполнении многих проектов часто объясняется нехваткой материалов. Если известно, что может возникнуть недостаток наличия материалов и это может сказаться на проекте, они должны быть включены в сетевой план проекта и должен быть составлен график.

Составление графика доставки и замены конструкций в течение этих 5 часов было чрезвычайно важным для управления проектом. Планирование материалов важно и при разработке продуктов, когда несвоевременное поступление товара на рынок может привести к потере доли на рынке.

Оборудование обычно представлено по типу, размеру и количеству. Для улучшения календарного планирования оборудование в некоторых случаях может быть взаимозаменяемо, но это не критично. Очень часто оборудование не рассматривают как ограничение. Наиболее распространенной ошибкой является то, что очень часто считают, что имеющихся ресурсов более чем достаточно для выполнения данного проекта.

Такой подход требует проверки наличия ресурсов для всех проектов и предусматривает резерв оборудования для конкретных потребностей проекта в будущем. Осознание до начала выполнения проекта того, что оборудование является фактором ограничения, поможет избежать авралов или издержек от задержки проекта.
Совокупность оборудования и людей называют рабочими центрами (РЦ). Это связано с тем, что для работы на оборудовании для выполнения технологических операций необходимы определенные навыки, которые характеризуются разрядом выполнения технологических операций.

Планирование изготовления деталей по РЦ достаточно раскрыто в статье [6], в которой представлены подход для формирования очередности изготовления и методология планирования выполнения технологических операций.

Задача планирования материалов рассматривается в статье [7]. Данное планирование основано на сроках изготовления изделий и потребностях в материалах в соответствии с технологическими процессами. Данные подходы не учитывают условия неопределенности, связанные с поставщиками и меняющейся структурой изделия. Для реализации данной задачи необходимо использовать вероятностные модели, основанные на ходе изготовления продукции ранее.

Таким образом, построение методики планирования материалов для обеспечения производства является одной из основных задач для изготовления продукции в срок.

\section{2 МоДЕЛЬ ОБЕСПЕЧЕНИЯ МАТЕРИАЛАМИ}

Пусть в плане по изготовлению множество изделий $I=\left\{I_{i}\right\}$, каждое изделие имеет характеристики $I_{i}=\left\{E_{j}, C_{j}, \operatorname{Pr}_{j}\right\}^{i}$, где $\left\{E_{j}\right\}$ - множество деталей, сборочных единиц (ДСЕ), $\left\{C_{j}\right\}$ - количество соответствующих деталей, $\left\{\operatorname{Pr}_{j}\right\}$ - подетальный порядок производства изделия.

Каждое ДСЕ, в свою очередь, имеет характеристики $E_{j}=\left\{R_{k}, T_{k}\right\}^{j} \cup\left\{M_{i}, C m_{i}\right\}^{j}$, где $\left\{R_{k}\right\}$ - множество РЦ, связанное со множеством операций, $\left\{T_{k}\right\}$ - нормативы времени на изготовление ДСЕ, $\left\{M_{i}\right\}$ - совокупность требуемых материалов, $\left\{C m_{i}\right\}$ - количества материалов.

Материалы можно разделить на классы:

1) основные, наличие которых требуется в начальной точке изготовления ДСЕ;

2) вспомогательные, их востребованность привязана к определенным операциям.

Для общности можно полагать, что основные материалы привязаны к комплектовочной операции.

Авторы статьи [6] предложили модель расчета очередности изготовления $E_{j}$ на основании длительности изготовления сборочных единиц $D E_{m}^{j}$, расстояния до вершины $R V_{p}^{j}$ и уровня вложенности $V_{p}^{j}$.

Так, длительность изготовления ДСЕ можно сформулировать следующим образом: 


$$
D E_{m}^{j}=P_{m}^{j} * \sum \frac{\sum D P_{m}^{j}}{\sum T_{k}},
$$

где $P_{m}^{j}$ - статистика закрытых технологических операций $O_{i}$,

$D P_{m}^{j}$ - длительность изготовления каждой технологической операции $O_{i}$.

Исходя из того, что каждая сборка $E_{j}$ имеет свое расстояние до вершины $R V_{p}^{j}$ и свой уровень вложенности $V_{p}^{j}$, очередь изготовления можно представить следующим образом:

$N E_{m}^{j}=\left|\frac{D E_{m}^{j}-\min \left(D E_{m}^{j}\right)}{\max _{L}\left(D E_{m}^{j}\right)-\min _{L}\left(D E_{m}^{j}\right)} * 99\right| * 100+V_{p}^{j}+1$.

После процесса распределения изготовления ДСЕ [8] по РЦ получаем для каждого ДСЕ множество $D^{j}=\left\{\left(D_{1}, D_{2}, O, R\right)_{i}\right\}$, где $R_{i}-$ Рц, $O_{i}$ - операция, выполняемая на данном РЦ в интервал времени $\left(D_{1_{i}}, D_{2_{i}}\right)$.

Абстрагируясь от РЦ, получаем временные интервалы выполнения операций $\left\{\left(D_{1}, D_{2}, O\right)^{i}\right\}$, а так как для каждой операции требуется набор материалов, то получаем $\left\{\left(D_{1}, D_{2}, M\right)^{k}\right\}$ - временные интервалы востребованности материалов.

Если $\exists i, j, k: \exists\left[D_{1}^{i}, D_{2}^{i}, M^{k}, C^{i}\right],\left[D_{1}^{j}, D_{2}^{j}, M^{k}, C^{j}\right]:$ $\left[D_{1}^{i}, D_{2}^{i}\right] \cap\left[D_{1}^{j}, D_{2}^{j}\right] \neq \varnothing$, то за обобщенный (укрупненный) интервал для материала $M^{k}$ принимаем $\left[\min \left(D_{1}^{i}, D_{2}^{j}\right), \max \left(D_{2}^{i}, D_{2}^{j}\right)\right]$ с общим количеством материала $C^{i}+C^{j}$.

Ставится задача определить необходимость закупки материалов на текущий момент времени со следующими показателями:

1) максимально допустимый срок закупки;

2) допустимый интервал объема партии;

3) допустимое множество поставщиков.

В структуре исходных данных имеем:

1) справочник материалов $\left\{M_{i}\right\}$, для каждого из которых определен интервал запаса $\left(C_{\min }^{i}, C_{\max }^{i}\right)$, pacсчитываемый по статистическим данным расхода материалов на производство за некоторый промежуток времени;

2) справочник поставщиков $\left\{P_{i}\right\}$, каждый из которых характеризуется показателями риска $\left\{\left(B_{r}, V_{r}, Z_{r}, S_{r}\right)^{i}\right\}$,где $B_{r}^{i}$ - зависимость количества брака от объема партии (может быть рассчитана как процент, либо как некоторая функция по статистическим данным), $V_{r}^{i}$ - минимально возможный объем партии для закупки; $Z_{r}^{i}=Z_{r_{1}}^{i}+Z_{r_{2}}^{i}$ - зависимость задержки поставки от объема партии $\left(Z_{r_{1}}^{i}\right.$ - планируемая поставщиком, $Z_{r_{2}}^{i}$ - незапланированная, рассчитывается из статистики поставок); $S_{r}^{i}$ - коэффициент завышения цены ( $n$ - общее количество поставщиков данного материала):

$$
S_{r}^{i}=\frac{\operatorname{Price}^{i}}{\frac{1}{n-1} \cdot \sum_{k=1, k \neq i}^{n} \text { Price }^{k}} .
$$

Для каждого материала $M^{k}$ имеем: интервал запаса $\left[C_{\min }^{k}, C_{\max }^{k}\right]$, требуемое для текущего изготовления общее количество $C_{M}^{k}$, остаток материала на складе $C_{r e s t}^{k}$. Определяем необходимость закупки и объем партии:

1) если $C_{\text {rest }}^{k}-C_{M}^{k} \in\left[C_{\min }^{k}, C_{\max }^{k}\right]$, то закупка не требуется;

2) если $0<C_{r e s t}^{k}-C_{M}^{k}<C_{\min }^{k} \quad$ то закупка второй очереди в количестве не менее, чем $C_{\min }^{k}-\left(C_{\text {rest }}^{k}-C_{M}^{k}\right)$;

3) если $C_{r e s t}^{k}-C_{M}^{k} \leq 0$, то закупка первой очереди в количестве не менее, чем $C_{\text {need }}^{k}$ :

$$
C_{\max }^{k}+C_{M}^{k} \geq C_{\text {need }}^{k} \geq \max \left(C_{M}^{k}-C_{\text {rest }}^{k}, C_{\min }^{k}\right) .
$$

\section{3 МЕТодИКА РЕШенИЯ ЗАДАЧИ И ПРАКТИЧЕСКАЯ РЕАЛИЗАЦИЯ}

Выбор наиболее подходящих поставщиков с минимальными показателями рисков может быть выполнен в соответствии с [9] и не входит в материалы данной работы.

Общая схема исследования по предлагаемой модели представлена на рисунке 2.

Схема базы данных (БД), полученная после загрузки и структуризации приведена на рисунке 3 .

Методы анализа временного ряда (ВР) и основные расчетные алгоритмы в формализованном виде приведены в [10]. Обобщенный порядок алгоритма анализа статистических данных с указанием номеров блоков программного кода приведен в таблице 1 .

1) Пример программного расчета аномальных точек по модифицированному критерию Ирвина:

\section{DECLARE @T_MAX INTEGER;}

DECLARE @I INTEGER;

SELECT @T_MAX=MAX(T) FROM STAT_MATERIAL; -WHERE ID_MATERIAL $=2$;

SELECT @I=2;

WHILE @I<=@T_MAX

BEGIN

SELECT M.ID,M.ID_MATERIAL,M.T,M.COUNT_USE-ML. COUNT_USE AS

MCOUNT_USE, 


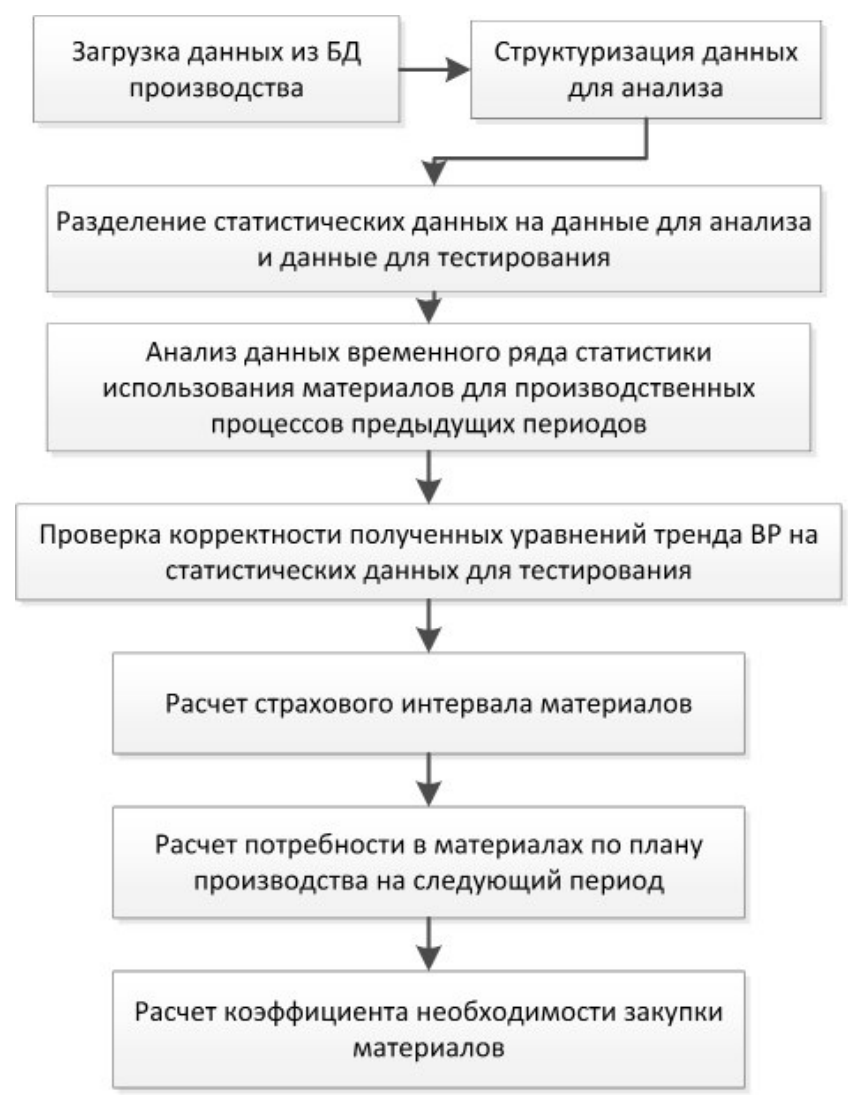

Рис. 2. Общая схема исследования

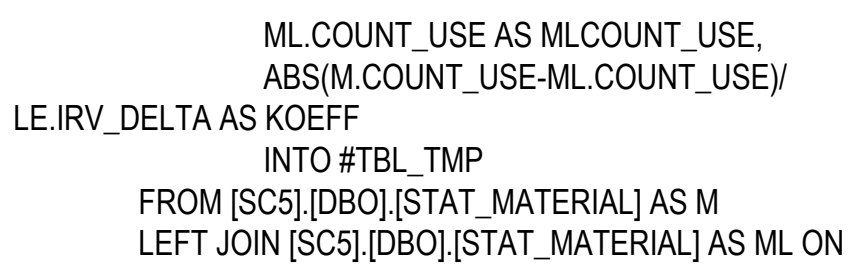

ML.ID_MATERIAL=M.ID_MATERIAL AND ML.T=<smiles>[CH]C</smiles>
SELECT MAX(T) FROM STAT_MATERIAL AS ST3 WHERE IRV_ANOMAL_MOD $=0$ AND ST3.ID_MATERIAL=M.ID_MATERIAL AND ST3.T<M.T ) LEFT JOIN [SC5].[DBO].[LIST_EXP] AS LE ON LE.ID_ MATERIAL=M.ID_MATERIAL

WHERE M.T=@I AND LE.IRV_DELTA $<>0$;

UPDATE STAT_MATERIAL SET IRV_ANOMAL_MOD=1 WHERE ID IN

(SELECT ID FROM \#TBL_TMP AS TT WHERE

TT.KOEFF>TT.STUD);

DROP TABLE \#TBL_TMP;

SELECT @I=@|+1;

END;

2) Сглаживание аномальных значений по методу среднеарифметической скользящей:

UPDATE STAT_MATERIAL SET COUNT_AVG=( SELECT SUM(COUNT_USE) FROM STAT_MATERIAL

AS ST

ID_MATERIAL) AND

WHERE (ST.ID_MATERIAL=STAT_MATERIAL.

$$
\begin{aligned}
& \text { (ST.T>=STAT_MATERIAL.T-2) AND (ST } \\
& \text { T<=STAT_MATERIAL.T+2))/( } \\
& \text { SELECT COUNT }\left(^{*}\right) \text { FROM STAT_MATERIAL AS ST } \\
& \text { WHERE (ST.ID_MATERIAL=STAT_MATERIAL. } \\
& \text { ID_MATERIAL) AND } \\
& \text { (ST.T>=STAT_MATERIAL.T-2) AND (ST. } \\
& \text { T<=STAT_MATERIAL.T+2)) }
\end{aligned}
$$

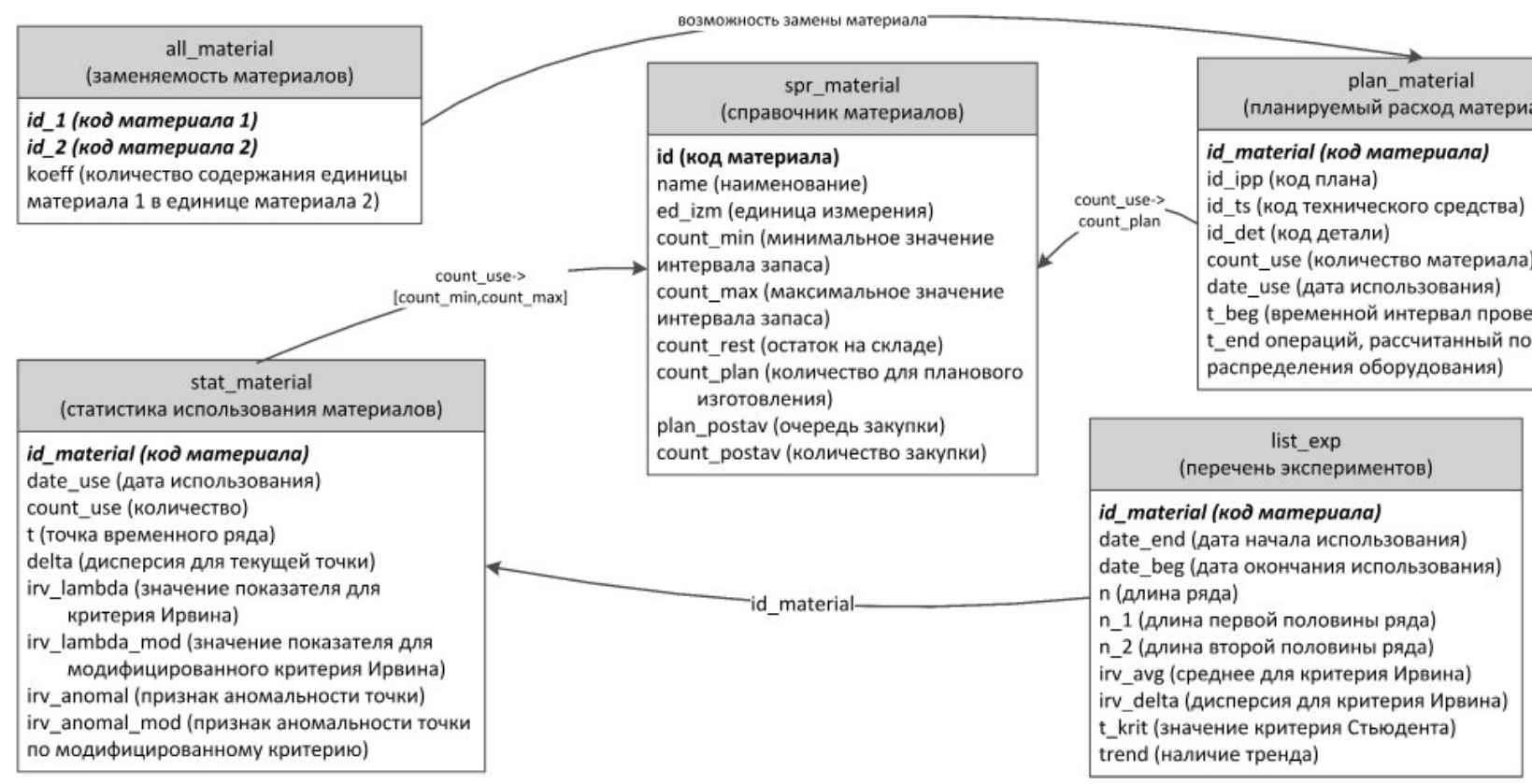

Рис. 3. Структура БД для расчетов по разработанной модели 
3) Проверка наличия тренда по методу однородности дисперсий с использованием справочника значений

$t$-критерия Стьюдента:

UPDATE LIST_EXP SET N_1=N/2;

UPDATE LIST_EXP SET N_2=N-N_1;

UPDATE LIST_EXP SET AVG_1=(SELECT AVG(COUNT_ USE) FROM STAT_MATERIAL AS

ST WHERE ST.ID_MATERIAL=LIST_EXP.ID_

MATERIAL AND ST.T<=LIST_EXP.N_1);

UPDATE LIST_EXP SET AVG_2=(SELECT AVG(COUNT_

USE) FROM STAT_MATERIAL AS

ST WHERE ST.ID_MATERIAL=LIST_EXP.ID_

MATERIAL AND ST.T<=LIST_EXP.N AND

ST.T>LIST_EXP.N_1);
UPDATE LIST_EXP SET DELTA_1=

(SELECT SUM((ST.COUNT_USE-LE.AVG_1)*(ST.

COUNT_USE-LE.AVG_1))

FROM STAT_MATERIAL AS ST

INNER JOIN LIST_EXP AS LE ON LE.ID_

MATERIAL=ST.ID_MATERIAL

WHERE ST.ID_MATERIAL=LIST_EXP.ID_MATERIAL

AND

ST.T<=LIST_EXP.N_1)/N_1;

UPDATE LIST_EXP SET DELTA_2=

(SELECT SUM((ST.COUNT_USE-LE.AVG_2)*(ST.

COUNT_USE-LE.AVG_2))

FROM STAT_MATERIAL AS ST

INNER JOIN LIST_EXP AS LE ON LE.ID_MATERIAL=ST.

ID_MATERIAL

Таблица 1

Основные этапы алгоритма анализа данных

\begin{tabular}{|c|c|c|}
\hline $\begin{array}{c}\text { Наименование } \\
\text { пункта }\end{array}$ & Основные формулы & $\begin{array}{c}\text { Номер } \\
\text { блока кода }\end{array}$ \\
\hline $\begin{array}{l}\text { Выявление аномальных } \\
\text { точек по обычному и моди- } \\
\text { фицированному критерию } \\
\text { Ирвина }\end{array}$ & $\lambda_{i}=\frac{\left|W_{i}-W_{i-1}\right|}{\sigma}, \sigma=\sqrt{\frac{\sum_{i=1}^{n}\left(W_{i}-\bar{W}\right)^{2}}{n-1}}, \bar{W}=\frac{1}{n} \sum_{i=1}^{n} W_{i}$ & 1 \\
\hline $\begin{array}{l}\text { Сглаживание ВР по мето- } \\
\text { дам среднеарифметиче- } \\
\text { ской скользящей, средне- } \\
\text { взвешенной скользящей, } \\
\text { экспоненциальному }\end{array}$ & $\begin{array}{l}\overline{W_{t}}=\frac{1}{2 m+1} \cdot \sum_{i=t-m}^{t+m} W_{i}, \overline{W_{t}}=\sum_{i=t-m}^{t+m} W_{i} \cdot v_{i} / \sum_{i=t-m}^{t+m} v_{i} \\
\overline{W_{t}}=\sum_{i=1}^{t} W_{i} \cdot v_{i} / \sum_{i=1}^{t} v_{i}, \quad v_{i}=\frac{1}{e^{T-t}}\end{array}$ & 2 \\
\hline $\begin{array}{l}\text { Проверка наличия тренда } \\
\text { ВР по методу проверки } \\
\text { однородности дисперсий } \\
\text { частей ряда с помощью } \\
\text { критерия Фишера }\end{array}$ & $\begin{array}{l}t=\frac{\left|\overline{W_{1}}-\overline{W_{2}}\right|}{\sigma \sqrt{\frac{1}{n_{1}}+\frac{1}{n_{2}}}, \overline{W_{l}}=\frac{1}{n_{l}} \sum_{k=1}^{n_{l}} W_{k},} \\
\sigma=\sqrt{\frac{\left(n_{1}-1\right) \sigma_{1}^{2}+\left(n_{2}-1\right) \sigma_{2}^{2}}{n_{1}+n_{2}-2}}, \sigma_{l}^{2}=\frac{1}{n_{l-1}} \sum_{k=1}^{n_{l}}\left(W_{k}-\overline{W_{k}}\right)^{2}\end{array}$ & 3 \\
\hline $\begin{array}{l}\text { Определение вида урав- } \\
\text { нения тренда с помощью } \\
\text { вычисления первого и вто- } \\
\text { рого среднего прироста }\end{array}$ & $\Delta Y_{t}=\frac{Y_{t+1}-Y_{t-1}}{2}, \Delta^{2} Y_{t}=\frac{\Delta Y_{t+1}-\Delta Y_{t-1}}{2}, \frac{\Delta Y_{t}}{Y_{t}}$ & 4 \\
\hline $\begin{array}{l}\text { Вычисление коэффициен- } \\
\text { тов уравнения тренда }\end{array}$ & $\left\{\begin{array}{l}a_{0} \cdot n+a_{1} \sum_{t=1}^{n} t=\sum_{t=1}^{n} W_{t} \\
a_{0} \sum_{t=1}^{n} t+a_{1} \sum_{t=1}^{n} t^{2}=\sum_{t=1}^{n} t \cdot W_{t}\end{array}\right.$ & 5 \\
\hline $\begin{array}{l}\text { Построение ВР } \\
\text { для тестового периода }\end{array}$ & $\widehat{W}_{t}=a_{0}+a_{1} \cdot t$ & 6 \\
\hline $\begin{array}{l}\text { Сравнение с тестовыми } \\
\text { данными }\end{array}$ & $E r r=\frac{1}{n} \sum_{t=1}^{n}\left(W_{t}-\widehat{W}_{t}\right)^{2}$ & 7 \\
\hline
\end{tabular}


WHERE ST.ID MATERIAL=LIST_EXP.ID_MATERIAL AND ST.T<=LIST_EXP.N

AND ST.T>LIST_EXP.N_1)/N_2;

UPDATE LIST_EXP SET T_KRIT=ABS(AVG_1-AVG_2)/ (DELTA_ST*SQRT(1/CAST(N_1 AS REAL)+1/

CAST(N_2 AS REAL))) WHERE N>2 AND DELTA_ST $<>0$;

UPDATE LIST_EXP SET TREND=1 WHERE T_KRIT>= (SELECT KOEFF FROM SPR_STUDENT AS SP

WHERE SP.N 1=

(SELECT TOP (1) N_1 FROM SPR_STUDENT AS SP2 WHERE SP2.N_1<=LIST_EXP.N_1 ORDER BY N_1

DESC)

AND SP.N_2=(SELECT TOP (1) N_2 FROM SPR_

STUDENT AS SP2

WHERE SP2.N_2<=LIST_EXP.N_2 ORDER BY N_2

DESC));

4) Для определения вида кривой тренда рассчитываются значения первого и второго прироста и их производные [11]:

UPDATE STAT_MATERIAL SET DELTA_1=ABS( ST WHERE

(SELECT COUNT_USE FROM STAT_MATERIAL AS

ST.ID_MATERIAL=STAT_MATERIAL.ID_MATERIAL

AND ST.T=STAT_MATERIAL.T-1)-

ST1 WHERE

(SELECT COUNT_USE FROM STAT_MATERIAL AS

ST1.ID_MATERIAL=STAT_MATERIAL.ID_MATERIAL

AND ST1.T=STAT_MATERIAL.T+1))/2;

UPDATE STAT_MATERIAL SET DERIV_1=DELTA_1/

COUNT_USE WHERE COUNT_USE $<>0$;

5) Как показали эксперименты (см. раздел «Эксперименты»), наиболее подходящим уравнением тренда является полином первого порядка $W_{t}=a_{0}+a_{1} \cdot t$, поэтому вычисление коэффициентов проводилось соответствующим образом из системы линейных уравнений, полученных по методу наименьших квадратов [11]:

INSERT INTO TBL_KOEFF (ID_MATERIAL,N,DATE_ BEG,DATE_END,MIN_T)

SELECT ID_MATERIAL,COUNT(*) AS N,MIN(DATE_ USE) AS DATE_BEG,

MAX(DATE_USE) AS DATE_END,MIN(T) AS MIN_T

FROM STAT_MATERIAL GROUP BY ID_MATERIAL;

UPDATE TK SET SUM_T=X.SUM_T,SUM_T_2=X.

SUM_T_2,SUM_Y=X.SUM_Y,SUM_T_Y=X.SUM_T_Y

FROM TBL_KOEFF TK JOIN (

SELECT ST1.ID_MATERIAL,SUM(ST1.T-TK2.

MIN_T+1) AS SUM_T,

AS SUM_T_2, SUM((ST1.T-TK2.MIN_T+1)*(ST1.T-TK2.MIN_T+1))

AS SUM T Y

SUM(COUNT_AVG_MOD) AS SUM_Y,

SUM((ST1.T-TK2.MIN_T+1)*COUNT_AVG_MOD)

FROM STAT_MATERIAL AS ST1
INNER JOIN TBL_KOEFF AS TK2 ON TK2.ID_

MATERIAL=ST1.ID_MATERIAL

AND ST1.DATE_USE >=TK2.DATE_BEG AND ST1.

DATE_USE<=TK2.DATE_END

GROUP BY ST1.ID_MATERIAL

) X ON X.ID_MATERIAL=TK.ID_MATERIAL.

\section{Расчет интервала запаса материала}

В качестве интервала страхового запаса $\left[C_{\min }^{k}, C_{\max }^{k}\right]$ принимаем:

1) $C_{\min }^{k}$ - минимальный расход за месяц для спрогнозированных данных. FROM (

SELECT MIN(X.SUM_MIN) AS SUM_MIN,X.ID_MATERIAL MONTH_MIN,

SELECT ID_MATERIAL,MONTH(DATE_USE) AS

SUM(COUNT_NEW) AS SUM_MIN

FROM TEST_2020

WHERE YEAR(DATE_USE) $=2020$

GROUP BY MONTH(DATE_USE),ID_MATERIAL) X

GROUP BY ID_MATERIAL);

2) $C_{\max }^{k}$ - максимальный расход за месяц для спрогнозированных данных. FROM

MONTH_MAX,

SELECT MAX(X.SUM_MAX) AS SUM_MAX,X.ID_MATERIAL SELECT ID_MATERIAL,MONTH(DATE_USE) AS

SUM(COUNT_NEW) AS SUM_MAX

FROM TEST_2020

WHERE YEAR(DATE_USE) $=2020$

GROUP BY MONTH(DATE_USE),ID_MATERIAL) X

GROUP BY ID_MATERIAL).

В качестве планового количества получаем количество материала, требуемое по плану изготовления, рассчитанному в соответствии с [8] по имеющимся данным на 2021 год.

1) сбор общих данных по плану 2021 года:

INSERT INTO PLAN_MATERIAL

(ID_MATERIAL,ID_TS,ID_DET,COUNT_USE,DATE_USE,T_ BEG,T_END,NUM_OPER)

SELECT X.ID,X.ID_TS,X.ID_DET,X.COUNT_

USE,NULL,0,0,X.ID_OPER

FROM (

SELECT MAT.ID,TB.TS AS ID_TS,TB.CODE AS ID_

DET,SP3998*TB.KOL AS

COUNT_USE,TB.ID_OPER AS ID_OPER

FROM TBL_MAT_OSN_PLAN AS TPLAN INNER JOIN TBL_OPER_STRUCT AS TB ON TPLAN.

OBJ_ID=TB.ID AND N_OPER='5' INNER JOIN SPR_MATERIAL AS MAT ON MAT.

ID_CHAR=TPLAN.TOV_IN AND

MAT.ED_IZM=TPLAN.SP4005

) $X$; 
2) подключение количества изготавливаемых технических средств (ТС) и начального времени изготовления каждого ДСЕ, полученного по алгоритмам [8]:

\section{UPDATE PLAN_MATERIAL SET COUNT_TS=( \\ SELECT COUNT $\left({ }^{*}\right)$ FROM TBL_TS \\ WHERE CODE_TS=PLAN_MATERIAL.ID_TS AND DATE_ OUT>='01.01.2021'); \\ UPDATE PLAN_MATERIAL SET DATE_USE $=($ \\ SELECT MIN(DATE_OUT) FROM TBL_TS WHERE CODE TS=PLAN_MATERIAL.ID_TS AND \\ DATE OUT >='01.01.2021'); \\ UPDATE PLAN_MATERIAL SET T_BEG $=($ \\ SELECT MIN(T_BEG) FROM TBL_RES WHERE NUM_ \\ OPER=PLAN_MATERIAL.NUM_OPER \\ AND TBL_RES.NUM_DET=PLAN_MATERIAL.ID_DET); \\ UPDATE PLAN_MATERIAL SET DATE \\ BEG=DATEADD(DAY,CAST(T_BEG/8 AS INT),DATE_USE);}

3) выборка общего количества планируемого расхода материалов по месяцам:

SELECT ID_MATERIAL,SUM(COUNT_USE*COUNT_TS), MONTH(DATE_BEG) AS MONTH_

FROM PLAN MATERIAL

GROUP BY ID_MATERIAL,MONTH(DATE_BEG)

ORDER BY ID_MATERIAL,MONTH(DATE_BEG).

\section{4 ЭКСПЕРИМЕНТАЛЬНЫЙ РАСЧЕТ}

Для проверки корректности построенной модели необходимо:

1. Подготовить статистические данные за предыдущие периоды и данные по планируемому изготовлению;

2. Провести экспериментальные расчеты по подготовленным данным;

3. Сделать выводы по пригодности модели к использованию в реальных процессах производства.

Для проведения статистических расчетов был взят состав из 771 единицы изделий с различными сроками изготовления и использованием более 2500 единиц различных материалов. В качестве данных для расчета тренда были взяты данные по расходу материалов за период с 01.09.2018 по 31.12.2019, для тестирования полученных уравнений - данные за период с 01.01.2020 по 31.12.2020. Для проведения расчетов по планируемому изготовлению были взяты данные по 773 единицам изделий со сроками изготовления с 12.01.2021 по 30.12.2021.

Были проведены расчеты аномальных точек по обычному и модифицированному критерию Ирвина, сглаживание аномальных точек по методам среднеарифметической скользящей, средневзвешенной по пяти точкам и экспоненциальному методу, вычисление наличия тренда по методу однородности дисперсий частей ряда.

В таблице 2 приведены данные по явному наличию тренда для исходного и сглаженных значений ряда.
Таблица 2

Статистика наличия тренда

\begin{tabular}{|l|c|c|c|}
\hline \multirow{2}{*}{$\begin{array}{l}\text { Значения } \\
\text { ряда }\end{array}$} & $\begin{array}{l}\text { Всего экс- } \\
\text { периментов }\end{array}$ & $\begin{array}{l}\text { Количество эксперимен- } \\
\text { тов с наличием тренда }\end{array}$ \\
\cline { 3 - 4 } & $\begin{array}{l}\text { обычный } \\
\text { критерий } \\
\text { Ирвина }\end{array}$ & $\begin{array}{l}\text { модифици- } \\
\text { рованный } \\
\text { критерий } \\
\text { Ирвина }\end{array}$ \\
\hline $\begin{array}{l}\text { Исходные } \\
\text { значения }\end{array}$ & 2027 & 305 & - \\
\hline $\begin{array}{l}\text { Среднеа- } \\
\text { рифметиче- } \\
\text { ский метод }\end{array}$ & 2027 & 471 & 444 \\
\hline $\begin{array}{l}\text { Средне- } \\
\text { взвешен- } \\
\text { ный метод }\end{array}$ & 2027 & 415 & 377 \\
\hline $\begin{array}{l}\text { Экспонен- } \\
\text { циальный } \\
\text { метод }\end{array}$ & 2027 & 399 & 345 \\
\hline
\end{tabular}

По результатам экспериментов лучше всего показал себя метод среднеарифметической скользящей с обычным критерием Ирвина. Дальнейшие расчеты проводились по значениям ряда, сглаженным данным методом.

Расчетные значения первого и второго прироста, а также их производных показали, что наиболее подходящие уравнения тренда - полином первого порядка с коэффициентами, рассчитанными отдельно для каждого квартала.

Используя полученные коэффициенты, было проведено моделирование значений ряда следующего периода (за 2020 год), полученные значения сравнены с имеющимися реальными значениями (получен ВР с количеством точек чуть менее 40000). Анализ ВР на цикличность и сезонность не проводился, так как на процесс закупки материалов данные показатели сильного влияния не оказывают. На рисунках 4, 5 приведены сравнительные графики значений расхода выборочных материалов (для иллюстрации выбраны два основных и два вспомогательных материала - из наиболее часто используемых и из среднеиспользуемых). В связи с большим количеством точек исходного ВР и принимая допущение, что закупка материалов планируется ежемесячно, для большей наглядности значения расхода материалов представлены не ежедневными показателями, а суммированными по месяцам.

В таблице 3 приведены основные расчетные коэффициенты и показатели для выборочных материалов.

По расчетным данным планового изготовления получаем значения общего расхода материала на планируемый месяц, представленные в таблице 4.

В таблице 5 представлен расчет значений планируемых закупок для одного из материалов (1661) при предположении, что изначально на складе материал отсутствует и закупаемый объем партии равен 100 единицам. 

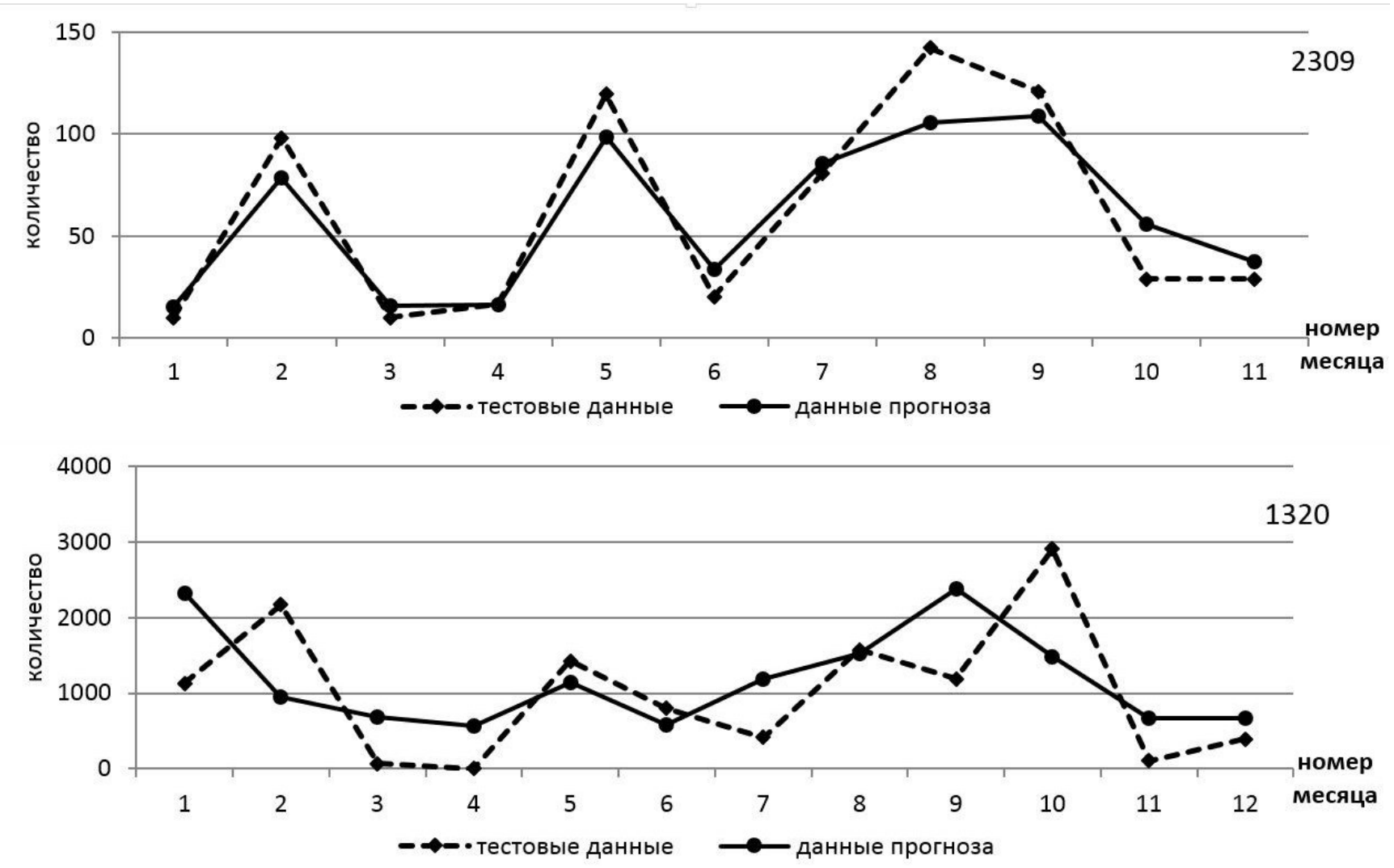

Рис. 4. Сравнительные графики значений ВР (основные материалы)
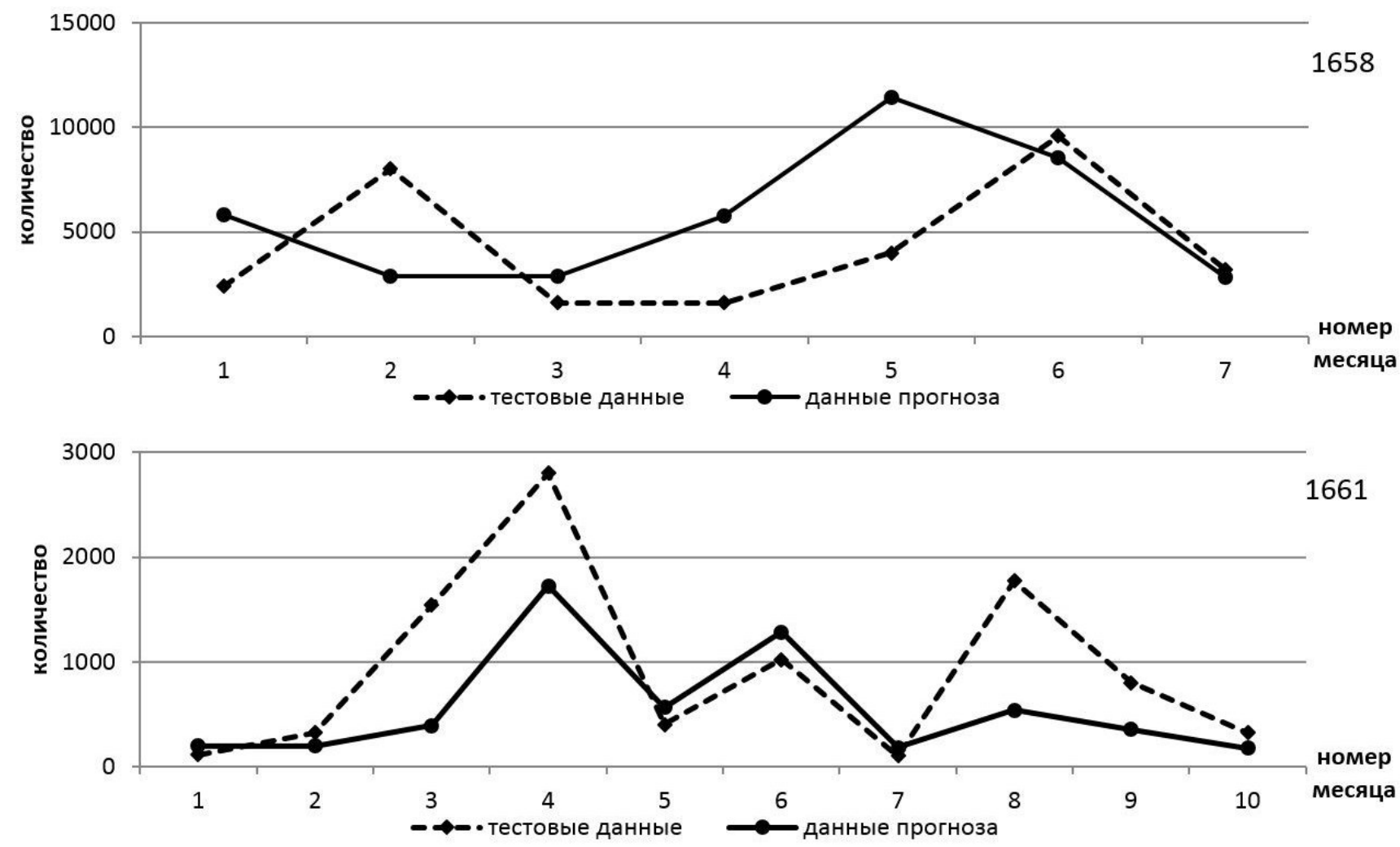

Рис. 5. Сравнительные графики значений ВР (вспомогательные материалы) 
Таблица 3

Расчетные коэффициенты для выборочных материалов

\begin{tabular}{|l|l|c|c|}
\hline \multicolumn{1}{|c|}{ Наименование материала (код) } & \multicolumn{1}{|c|}{ Уравнение тренда } & $C_{\min }$ & $C_{\max }$ \\
\hline Лист АМг5М 8х1200х3000 (1320) & $y=0,471 \cdot t+124,458$ & 258,72 & 1900,734 \\
\hline Круг 10,0-һ9 ГОСТ 7417-75/ 12Х18Н10Т ГОСТ 5949-2018 (2309) & $y=0,0935 \cdot t+15,311$ & 18,14 & 108,657 \\
\hline Каучук Низкомолекулярный ПДИ-3АК ТУ38-103410-78 (1658) & $y=-5,713 \cdot t+2912,485$ & 1749,21 & 7062,753 \\
\hline Газ аргон, в/с (1661) & $y=-0,764 \cdot t+198,606$ & 212,839 & 1957,664 \\
\hline
\end{tabular}

Таблица 4

Значения общего расхода материала на планируемый месяц

\begin{tabular}{|c|c|c|c|c|c|c|c|c|c|c|c|c|}
\hline \multirow{2}{*}{$\begin{array}{c}\text { Код } \\
\text { материала }\end{array}$} & \multicolumn{12}{|c|}{ № месяца } \\
\hline & 1 & 2 & 3 & 4 & 5 & 6 & 7 & 8 & 9 & 10 & 11 & 12 \\
\hline 1320 & 33,95 & 10,8 & 47,8 & 488,45 & 243,87 & 65,65 & 11,62 & 4,96 & 4,584 & 14,3 & 7,07 & 5,786 \\
\hline 2309 & 10,05 & 98,01 & 10,05 & 16,34 & 119,38 & 20,11 & 80,42 & 142 & 120,63 & 28,9 & 28,9 & - \\
\hline 1658 & 2400 & 800 & 1600 & - & - & - & - & 1600 & 4000 & 9600 & - & 3200 \\
\hline 1661 & 110 & 320 & 1540 & 2800 & 400 & 1020 & 100 & 1770 & 800 & 320 & - & - \\
\hline
\end{tabular}

Таблица 5

Расчет значений планируемых закупок

\begin{tabular}{|c|c|c|c|c|c|}
\hline № месяца & $\begin{array}{c}\text { Остаток } \\
\text { на складе }\end{array}$ & $\begin{array}{c}\text { Планируемый } \\
\text { расход }\end{array}$ & \multicolumn{2}{|c|}{ Интервал закупки } & Объем закупки \\
\hline 01.2021 & 0 & 110 & 213 & 2068 & 300 \\
\hline 02.2021 & 190 & 320 & 213 & 2278 & 300 \\
\hline 03.2021 & 170 & 1540 & 1370 & 3497 & 1400 \\
\hline 04.2021 & 30 & 2800 & 2770 & 4758 & 2800 \\
\hline 05.2021 & 60 & 400 & 340 & 2358 & 400 \\
\hline 06.2021 & 120 & 1020 & 900 & 2978 & 900 \\
\hline 07.2021 & 0 & 100 & 213 & 2058 & 300 \\
\hline 08.2021 & 200 & 1770 & 1570 & 3728 & 1600 \\
\hline 09.2021 & 230 & 800 & 570 & 2758 & 600 \\
\hline 10.2021 & 30 & 320 & 290 & 2278 & 300 \\
\hline
\end{tabular}

\section{ЗАКЛЮЧЕНИЕ}

По результатам проведенного исследования можно сделать следующие выводы:

1) разработана модель обеспечения производства материальными ресурсами на основании статистики расхода материалов за предыдущие периоды и очередности изготовления продукции;

2) разработана методика расчета анализа ВР и приведены этапы алгоритма;

3) сделаны выводы об оптимальных способах сглаживания данных, модификации метода выявления аномальных точек, о возможных видах тренда в различных временных периодах;

4) разработана структура БД, реализованы все стандартные и модифицированные алгоритмы;

5) проведены численные эксперименты на реальных данных за 2018-2019 годы, определены оптимальные коэффициенты уравнений тренда, на основании результатов вычислений проведено моделирование значений ВР на 2020 год, проведено сравнение с реальными данными, которое позволяет говорить о допустимости применения рассмотренных методов к исследуемым данным.

Таким образом, следует сказать, что предложенная методика позволяет спрогнозировать планируемый расход материалов без существенных доработок используемой системы автоматизации планирования производственной деятельности.

\section{СПИСОК ЛИТЕРАТУРЫ}

1. Рыжов Е.В., Бахотский В.В. Организация и планирование производства : учеб. пособие. Псков : Псковский государственный университет, 2013. 148 с. 
2. Казаченко Л.Д. Развитие современных систем управления предприятием // Вестник ЧитГУ. 2010. № 7. C. $16-22$.

3. Кобец Е.А. Планирование на предприятии: учеб. пособие. Таганрог : ТРТУ, 2006. 130 с.

4. Смагина М.В., Грошева И.А. Резервы повышения качества стратегического планирования на промышленных предприятиях // Вестник Саратовского ГСЭУ. 2011. № 4. С. 110-113.

5. Черкасова О.В. Управление предприятием (организацией) : учеб. пособие. Саранск : МГУ, 2010. 212 с.

6. Перцев А.А., Подобрий А.Н. Формирование и анализ плана производства машиностроительного предприятия // Автоматизация процессов управления. 2019. № 1 (55). С. 74-83.

7. Перцев А.А., Подобрий А.Н. Подход к автоматизированному формированию плана изготовления производственного подразделения проектной организации // Сб. науч. тр. 7-й Всерос. науч.-техн. конф. аспирантов, студентов и молодых ученых «Информатика и вычислительная техника» (ИВТ-2015), г. Ульяновск, 25-27 мая 2015. Ульяновск : УлГТУ, 2015. С. 398-405.

8. Перцев А.А., Подобрий А.Н., Радионова Ю.А. Реализация равномерной загрузки производства машиностроительного предприятия // Автоматизация процессов управления. 2020. № 4 (62). С. 49-60.

9. Емельянов А.А., Радионова Ю.А., Савкин А.Л. Моделирование и статистический контроль рисков процесса закупки // Автоматизация процессов управления. 2017. № 2 (48). С. 49-59.

10. Татаренко С.И. Методы и модели анализа временных рядов : метод. указания к лаб. работам. Тамбов : Изд-во Тамб.гос. техн. ун-та, 2008. 32 с.

11. Радионова Ю.А., Савкин А.Л. Построение модели прогноза пиковых нагрузок рабочего процесса на основе анализа временного ряда // Автоматизация процессов управления. 2020. № 2 (60). С. 53-61.

\section{REFERENCES}

1. Ryzhov E.V., Bakhotskii V.V. Organizatsiia $i$ planirovanie proizvodstva. Ucheb. posobie [Organization and Production Planning. Tutorial]. Pskov, Pskov State University Publ., 2013. 148 p.

2. Kazachenko L.D. Razvitie sovremennykh sistem upravleniia predpriiatiem [Development of Modern Enterprise Management Systems]. Vestnik ChitGU [Bull. of Chita State University], 2010, no. 7, pp. 16-22.
3. Kobets E.A. Planirovanie na predpriiatii. Ucheb. posobie [Enterprise Planning. Tutorial]. Taganrog, TRTU Publ., 2006. 130 p.

4. Smagina M.V., Grosheva I.A. Rezervy povysheniia kachestva strategicheskogo planirovaniia na promyshlennykh predpriiatiiakh [Potential for Quality Improvement of Strategic Planning atIndustrial Enterprise]. Vestnik Saratovskogo GSEU [Proc. of Saratov State Social and Economic University], 2011, no. 4, pp. 110-113.

5. Cherkasova O.V. Upravlenie predpriiatiem (organizatsiei). Ucheb. posobie [Factory Management. Tutorial]. Saransk, MGU Publ., 2010. 212 p.

6. Pertsev A.A., Podobrii A.N. Formirovanie $\mathrm{i}$ analiz plana proizvodstva mashinostroitelnogo predpriiatiia [Production Planning of the Machine-Building Enterprise]. Avtomatizatsiia protsessov upravlenia [Automation of Control Processes], 2019, no. 1 (55), pp. 74-83.

7. Pertsev A.A., Podobrii A.N. Podkhod k avtomatizirovannomu formirovaniiu plana izgotovleniia proizvodstvennogo podrazdeleniia proektnoi organizatsii [An Approach to Automated Plan Formation of a Production Unit in Project Organization].Sb. nauch. tr. 7-iVseros. nauch.tekhn. konf. aspirantov, studentov i molodykh uchenykh "Informatika i vychislitelnaia tekhnika" (IVT-2015) [Proc. of 7th Russian Sci.-Tech. Conf. on Informatics and Computer Facilities for Postgraduate, Students, and Young Scientists (IVT-2015]. Ulyanovsk, UISTU Publ., 2015, pp. 398-405.

8. Pertsev A.A., Podobrii A.N., Radionova lu.A. Realizatsiia ravnomernoi zagruzki proizvodstva mashinostroitelnogo predpriiatiia [The Balanced Production Load of a Machine-Engineering Organization]. Avtomatizatsiia protsessov upravleniia [Automation of Control Processes], 2020, no. 4 (62), pp. 49-60.

9. Emelianov A.A., Radionova lu.A., Savkin A.L. Modelirovanie i statisticheskii kontrol riskov protsessa zakupki [Simulation and Statistical Control of Procurement Risks]. Avtomatizatsiia protsessov upravleniia [Automation of Control Processes], 2017, no. 2 (48), pp. 49-59.

10. Tatarenko S.I. Metody i modeli analiza vremennykh riadov. Metod. ukazaniia k lab. rabotam [Methods and Models for Analysing the Time Series. Lab. Study Guide]. Tambov, Tamb. State Tech. Un. Publ., 2008. 32 p.

11. Radionova lu.A., Savkin A.L. Postroenie modeli prognoza pikovykh nagruzok rabochego protsessa na osnove analiza vremennogo riada [Forecast Modeling of Peak Work Loads based on Time Series Analysis]. Avtomatizatsiia protsessov upravleniia [Automation of Control Processes], 2020, no. 2 (60), pp. 53-61. 